\title{
TERAHERTZ QUANTUM BEATS IN MOLECULAR LIQUIDS
}

\author{
R. LEONHARDT, W. HOLZAPFEL, W. ZINTH and W. KAISER \\ Physik Department E11, Technische Universität München, D-8000 Munich 2, West Germany
}

Received 1 December 1986

\begin{abstract}
With ultrashort pulses of less than $100 \mathrm{fs}$ it is possible to excite coherently several vibrational modes of polyatomic molecules simultaneously. The femtosecond time resolution of the experiment allows the study of pronounced high-frequency beat phenomena up to $10 \mathrm{THz}$. The frequency difference between vibrational modes separated by more than $300 \mathrm{~cm}^{-1}$ may be determined with high precision.
\end{abstract}

\section{Introduction}

During the past decade, time-resolved coherent Raman investigations on the time scale of $10^{-12} \mathrm{~s}$ have received considerable attention. With picosecond laser pulses coherent vibrational excitations of a larger number of molecular modes were investigated [1-3]. Much valuable information came from these studies. For instance, vibrational decay processes were elucidated [2-4], the resolution in congested spectral regions was improved [5-7], and beating of neighboring vibrations was observed $[4,7,8]$.

In this Letter the application of femtosecond pulses to coherent Raman spectroscopy of molecular transitions in liquid systems is discussed. Femtosecond pulses allow us to investigate the decay dynamics of vibrational transitions with dephasing times $T_{2}$ of less than $1 \mathrm{ps}$ and provide the possibility to study fast beating phenomena on a frequency scale of many terahertz. Here, we focus on the latter topic. Several vibrational modes of the same or of different molecules are simultaneously excited by a driving force of approximately $100 \mathrm{fs}\left(10^{-13} \mathrm{~s}\right)$ duration. Strong beating phenomena are observed which allow the frequency differences between various modes to be determined with high precision. The high time resolution of the present system permits the observation of beat frequencies of approximately $10 \mathrm{THz}\left(10^{13}\right.$ $\mathbf{s}^{-1}$ ).

\section{Theory}

Time-resolved coherent Raman scattering is commonly treated under the following assumptions: The light fields are described by Maxwell's equations and the vibrational transitions are represented by twolevel systems. The vibrational excitation of an ensemble of molecules is described by the expectation value of the vibrational amplitude $\langle q\rangle=$ $\frac{1}{2} \mathrm{i} Q \exp \left(-\mathrm{i} \omega_{q} t+\mathrm{i} k_{q} x\right)+$ c.c. One assumes that the excitation is weak. We limit the discussion to polarized Lorentzian-shaped Raman lines. Under these conditions the different vibrational modes are independently excited. The total vibrational excitation may be described as the superposition of the individual states $\left\langle q_{j}\right\rangle$ with well defined amplitudes $Q_{j}$, frequencies $\omega_{q}$, and phase factors $\phi_{\text {: }}$ :

$$
\begin{aligned}
& \langle q\rangle=\sum_{j}\left\langle q_{j}\right\rangle \\
& \quad=\frac{1}{2} \mathrm{i} \sum_{j} Q_{j} \exp \left(-\mathrm{i} \omega_{\mathcal{q}} t+\mathrm{i} k_{q} x+\mathrm{i} \phi_{j}\right)+\text { c.c. }
\end{aligned}
$$

The coherent amplitude of a Raman-active mode with frequency $\omega_{q j}$ can be excited via transient stimulated Raman scattering [9] by a pair of light pulses, the laser pulse $E_{\mathrm{L}}$, and the Stokes pulse $E_{\mathrm{S}}$. The electric fields are considered to be plane waves, e.g. $E_{\mathrm{L}}=\frac{1}{2} E_{\mathrm{L}} \exp \left(-\mathrm{i} \omega_{\mathrm{L}} t+\mathrm{i} k_{\mathrm{L}} x\right)+$ c.c. with the wave vector $k_{\mathrm{L}}$ and frequency $\omega_{\mathrm{L}}$ for the laser field. The driving force $F(x, t)$ for the coherent amplitude $Q$ is 
proportional to the product of the laser and Stokes fields, $F(x, t) \propto E_{\mathrm{L}} E_{\mathrm{S}}^{*}$. The carrier frequency of the excitation force is $\omega_{\mathrm{L}}-\omega_{\mathrm{S}}$. The application of linear response theory to the excitation process leads to the following equation for the coherent amplitude $Q_{j}[1]$ :

$$
\begin{gathered}
Q_{J} \exp \left(\mathrm{i} \phi_{J}\right)=\kappa \int_{-\infty}^{t} \mathrm{~d} t^{\prime} E_{\mathrm{L}}\left(t^{\prime}\right) E_{\mathrm{S}}^{*}\left(t^{\prime}\right) \\
\times \exp \left[\left(t^{\prime}-t\right) / T_{2,}\right] \exp \left[-\mathrm{i} \Delta \omega_{,} t^{\prime}\right] .
\end{gathered}
$$

The constant $\kappa$, is defined in ref. [1] and contains material parameters such as the Raman cross section and vibrational frequency $\omega_{q v} . T_{2 j}$ stands for the dephasing time of the transition $j$. The detuning between excitation frequency and vibrational mode is given by $\Delta \omega_{j}=\omega_{L}-\omega_{S}-\omega_{q j}$. On account of the large bandwidth of the ultrashort driving force, several modes may be excited simultaneously.

In a time-resolved coherent Raman experiment the coherent amplitude $\langle q\rangle$ which is generated at time zero is monitored by coherent Raman scattering of a delayed probing pulse $E_{\mathrm{L}_{2}}$. We investigate the antiStokes part of the spectrum, i.e. we observe light with field amplitude $E_{\mathrm{AS}}$ generated according to

$$
\begin{array}{r}
E_{\mathrm{AS}}\left(t_{\mathrm{D}}, t\right) \propto \sum_{J} \int_{0}^{L} \mathrm{~d} x Q_{J}(t, x) E_{\mathrm{L}_{2}}\left(t-t_{\mathrm{D}}, x\right) \\
\quad \times \exp \left[-\mathrm{i}\left(\omega_{\mathrm{L}_{2}}+\omega_{q \jmath}\right) t+\mathrm{i} \Delta k_{J} x+\mathrm{i} \phi_{J}\right] .
\end{array}
$$

Here, $\Delta k_{\jmath}=k_{\mathrm{L}_{2}}+k_{q}-k_{\mathrm{AS} \text {, }}$ determines the spatial phase mismatch of the $j$ th component. Using a suitable beam geometry (phase matching) $[1,4]$ the values of $\Delta k$, may be minimized for the different modes. With a sufficiently short interaction length $L$, $L<1 / \Delta k_{j}$, the small remaining mismatch $\Delta k_{j}$ does not influence the recorded signal $E_{\mathrm{AS}}$. Experimentally, one observes the scattered energy $S_{\mathrm{AS}}$ at the end of the sample, which is proportional to the time-integrated intensity, i.e. $S_{\mathrm{AS}}\left(t_{\mathrm{D}}\right) \propto \int \mathrm{d} t\left|E_{\mathrm{AS}}\left(t, t_{\mathrm{D}}\right)\right|^{2}$.

For sufficiently short exciting and probing pulses, $t_{\mathrm{p}} \ll T_{2}$, and for late delay times, $t_{\mathrm{D}} \gg t_{\mathrm{p}}$, the salient features of the coherent signal are: (i) For a single vibrational mode the signal decays exponentially with $\exp \left(-2 t_{\mathrm{D}} / T_{2}\right)$. (ii) For several simultaneously excited transitions the signal decay is more complex. The coherently scattered light field consists of a sum over contributions originating from the different vibrational modes $j$ :

$$
S_{\mathrm{AS}}\left(t_{\mathrm{D}}\right) \propto\left|\sum_{J} Q_{l}\left(t_{\mathrm{D}}\right) \exp \left(-\mathrm{i} \omega_{q} t_{\mathrm{D}}+\mathrm{i} \phi_{j}\right)\right|^{2} .
$$

The evolution of the coherent signal is readily demonstrated for the case of two molecular transitions at frequencies $\omega_{q}$ and $\omega_{q}+\delta \omega$. For the time dependence of the coherent amplitude $Q_{J}=A_{j} \exp \left(-t / T_{2}\right)$ with $j=1,2$. The coherent signal is written in the form

$$
\begin{aligned}
& S_{\mathrm{AS}}\left(t_{\mathrm{D}}\right) \propto A_{1}^{2} \exp \left(-2 t_{\mathrm{D}} / T_{21}\right) \\
& \quad \times\left\{1+\left(A_{2}^{2} / A_{1}^{2}\right) \exp \left[-2 t_{\mathrm{D}}\left(T_{22}^{-1}-T_{21}^{-1}\right)\right]\right. \\
& \quad+2\left(A_{2} / A_{1}\right) \cos \left(\delta \omega t_{\mathrm{D}}+\phi_{1}-\phi_{2}\right) \\
& \left.\quad \times \exp \left[-t_{\mathrm{D}}\left(T_{22}^{-1}-T_{21}^{-1}\right)\right]\right\} .
\end{aligned}
$$

We assume $T_{21}>T_{22}$. From eq. (5) it is evident that the coherent signal consists of a sum of three contributions multiplied by the factor $\exp \left(-2 t_{\mathrm{D}} T_{21}^{-1}\right)$ (the exponential decay can be removed from measured coherent data by multiplication with $\left.\exp \left(2 t_{\mathrm{D}} T_{21}^{-1}\right)\right)$. A constant part, a term decaying with the difference of the two decay rates, and - most interesting - a contribution oscillating at the frequency difference $\delta \omega$ are found. This oscillating part decays with $\exp \left[-t_{\mathrm{D}}\left(T_{22}^{-1}-T_{21}^{-1}\right)\right]$. The corresponding observation time for which the oscillations may be followed is $T=T_{22} T_{21} /\left(T_{21}-T_{22}\right)$. For similar dephasing times $T_{21} \approx T_{22}$ the time $T$ is much longer than the dephasing times $T_{2 j}$. In most coherent Raman experiments the decay of the coherent signal is observed over many decay times, i.e. one may follow the oscillations between the two modes over the entire observation time $T$. Thus the frequency difference $\delta \omega$ may be determined more accurately than by spontaneous Raman measurements. We recall that in spontaneous Raman spectroscopy the precision of the frequency position is determined by the bandwidth given by $\Delta \nu=\left(\pi T_{2 j}\right)^{-1}$.

\section{Experimental}

Our experimental system is depicted schematically in fig. 1. Pulses from a mode-locked argon-ion laser synchronously pump two dye lasers. The spe- 


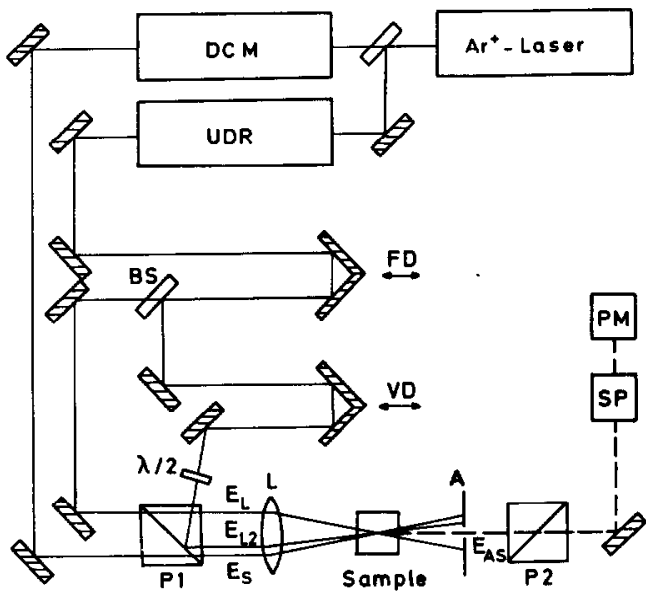

Fig. 1. Schematic of the experimental system used to measure terahertz beat frequencies by time-resolved coherent Raman scattering. A mode-locked argon-ion laser pumps a femtosecond unidirectional ring dye laser (UDR) and a picosecond dye laser DCM. Fixed and variable delay lines, FD and VD, polarizer P1 and $P 2$, beamsplitter BS, lens $L$, aperture A, spectrometer SP, photomultiplier PM.

cially designed dye laser UDR [10], a unidirectional ring dye laser, contains an amplifying jet (dye rhodamine 6G) and an absorber jet (dye DODCI). This laser emits pulses with a duration of approximately $80 \mathrm{fs}$ at $\lambda=625 \mathrm{~nm}$. The pulses from this laser provide the exciting and probing light fields $E_{\mathrm{L}}$ and $E_{\mathrm{L}_{2}}$, respectively with an average power of $\approx 10 \mathrm{~mW}$. The second laser, DCM, is a standard synchronously mode-locked picosecond dye laser with an amplifying dye DCM and a three-plate birefringent filter as a tuning element (power $30 \mathrm{~mW}$ ). This laser may be tuned between $\lambda=640$ and $690 \mathrm{~nm}$, emitting pulses $E_{\mathrm{S}}$ at the Stokes frequency of $6 \mathrm{ps}$ duration. The length of the unidirectional ring laser is actively controlled in order to synchronize the pulses from the femtosecond and the picosecond laser with temporal jitter of less than $5 \mathrm{ps}$. Part of the femtosecond pulses from the UDR laser $\left(E_{\mathrm{L}}\right)$ and the pulses from the DCM laser $\left(E_{\mathrm{S}}\right)$, both having parallel polarisation, serve to excite the molecular vibrations in the sample. It is evident from eq. (2) that the product $E_{\mathrm{L}} E_{\mathrm{S}}$ determines the driving force. This driving force exists only for the time duration of the shorter of the two pulses, i.e. for the time of $80 \mathrm{fs}$. A major advantage of the system is the ready tunability of the excitation frequency $\omega_{L}-\omega_{S}$ using the birefringent filter of the picosecond laser.

The coherent vibrational excitation is monitored by probing pulses $E_{\mathrm{L}_{2}}$ generated by the UDR laser with the help of the beam splitter BS. These pulses are polarized perpendicular to the excitation pulses. The three beams are focused into the sample using a geometry appropriate for proper anti-Stokes phase matching. The anti-Stokes radiation $E_{\mathrm{AS}}$ generated by the probing process passes the second polarizer and is detected after the broad-band spectrometer SP by the cooled photomultiplier PM. The coherent signal $S_{\mathrm{AS}}$ is recorded as a function of the delay time (set by the optical delay line VD) between the exciting and probing pulses $E_{\mathrm{L}}$ and $E_{\mathrm{L}_{2}}$, respectively. It should be repeated that the time resolution of the apparatus is only determined by the short pulses of the femtosecond laser. The jitter between the femtosecond and the picosecond laser influences the signal amplitude but does not affect the time resolution.

\section{Results and discussion}

\subsection{Pyridine}

Liquid pyridine has two Raman-active vibrations at frequencies 991 and $1030 \mathrm{~cm}^{-1}$. They are assigned to two $A_{1}$ ring modes [11]. Both vibrations have similar spectral widths $\left(\Delta \nu \approx 2.2 \mathrm{~cm}^{-1}\right)$ and similar Raman cross sections; they are separated by $39 \mathrm{~cm}^{-1}$. In the Raman excitation process a frequency difference of $\left(\omega_{\mathrm{L}}-\omega_{\mathrm{S}}\right) / 2 \pi c \approx 1010 \mathrm{~cm}^{-1}$ between the laser and the Stokes frequencies is applied. Due to the broad spectral widths of the femtosecond exciting force of $\Delta \omega / 2 \pi c>200 \mathrm{~cm}^{-1}$ both pyridine modes are simultaneously excited in the experiment. Fig. 2 shows the observed anti-Stokes signal plotted as a function of the time delay between exciting and probing pulses. During the excitation process, at time zero, the coherent signal rises to a pronounced peak. It subsequently decays quickly over more than an order of magnitude. Later, the signal recovers and shows strong oscillations. The modulation depth exceeds a factor of ten. Two features of the coherent signal are of special interest here:

(i) The period of oscillation is $0.85 \pm 0.01 \mathrm{ps}$. Consequently, the frequency difference between the two modes is $1.175 \pm 0.015 \mathrm{THz}$ or $\delta v / c=39.2 \pm 0.5$ 


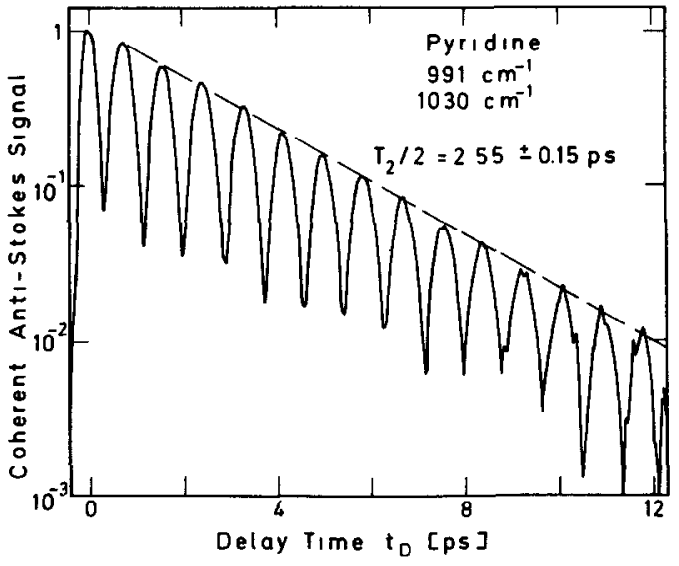

Fig. 2. Coherent anti-Stokes Raman signal obtained for liquid pyridine. The time-resolved data exhibit the strong beating between two vibrational modes of pyridine at 991 and $1030 \mathrm{~cm}^{-1}$. The frequency difference can be accurately determined to be $39.2 \pm 0.5 \mathrm{~cm}^{-1}$.

$\mathrm{cm}^{-1}$. We emphasize that the beat frequency may be determined with high precision depending essentially on the signal-to-noise ratio of the experiment and the precision of the optical delay line.

(ii) The envelope of the oscillation peaks decays exponentially with the decay time $\frac{1}{2} T_{2}=2.55 \pm 0.15$ ps. The depth of the oscillations slightly decreases for longer observation times, indicating a slight difference of $\approx 20 \%$ between the dephasing times of the two modes.

\subsection{Cyclohexane:benzene}

In fig. 3 we show the decay of the coherent antiStokes signal recorded from the binary mixture of cyclohexane and benzene of $1: 1$ by volume. The frequency of the exciting force was set to $900 \mathrm{~cm}^{-1}$ in order to excite simultaneously Raman-active transitions in cyclohexane at $802 \mathrm{~cm}^{-1}$ and in benzene at $992 \mathrm{~cm}^{-1}$. In fig. 2 one observes an enhanced signal peak at time zero and a subsequent oscillatory behavior. The fast oscillations clearly demonstrate that it is possible to excite coherently vibrational modes which are separated by $190 \mathrm{~cm}^{-1}$. From the beating period we can determine the frequency difference $\delta \nu$ of the two vibrational modes in the solution to be $\delta \nu / c=189.7 \pm 1.5 \mathrm{~cm}^{-1}$ or $\delta \nu=5.7 \mathrm{THz}$. In the present experiment the accuracy of $\Delta \nu$ is determined by the signal-to-noise ratio. Additional inter-

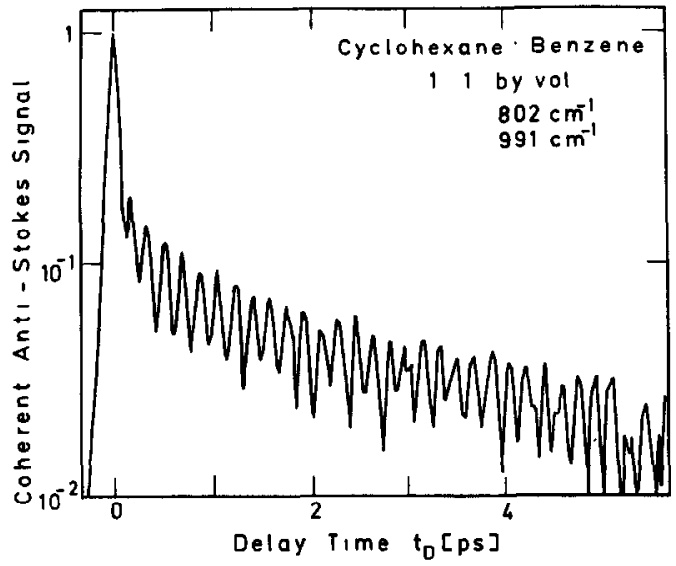

Fig. 3. Coherent anti-Stokes Raman signal for the mixture of cyclohexane and benzene. Two modes separated by $\approx 189 \mathrm{~cm}^{-1}$ are excited simultaneously, leading to the observed beat frequency of $5.7 \mathrm{THz}$.

esting features are seen at early delay times. The signal peak at time zero is strongly enhanced and at delay time $t_{\mathrm{D}}<1 \mathrm{ps}$ the signal decay is faster $(\tau \approx 0.8 \mathrm{ps})$ than at later delay times. The two observations may be explained as follows. (i) The vibrational modes of benzene and cyclohexane at 991 and $802 \mathrm{~cm}^{-1}$ are located in the wings of the spectrum of the exciting force. They are only weakly excited. As a result, it is possible to observe contributions from the non-resonant nonlinear susceptibility $\chi_{\mathrm{NR}}^{(3)}$; the latter enhances the signal at time zero [12]. (ii) In addition to the two strong modes at 802 and $992 \mathrm{~cm}^{-1}$, a weaker but broader mode exists in cyclohexane at $1030 \mathrm{~cm}^{-1}$. This third mode with small $T_{2}$ may cause the more rapid decay of the signal at early delay times.

We have performed experiments with the same mixture of cyclohexane and benzene, applying different excitation wavelengths. When the driving force is tuned away from the center between the two major modes the modulation depth decreases. An exponential decay was observed, when the driving frequency was close to the resonance frequencies of either benzene or cyclohexane.

\subsection{Cyclohexane:pyridine}

With the same excitation frequency of $900 \mathrm{~cm}^{-1}$ as used in the experiment of fig. 3 , we find a completely different scattering pattern when working with 


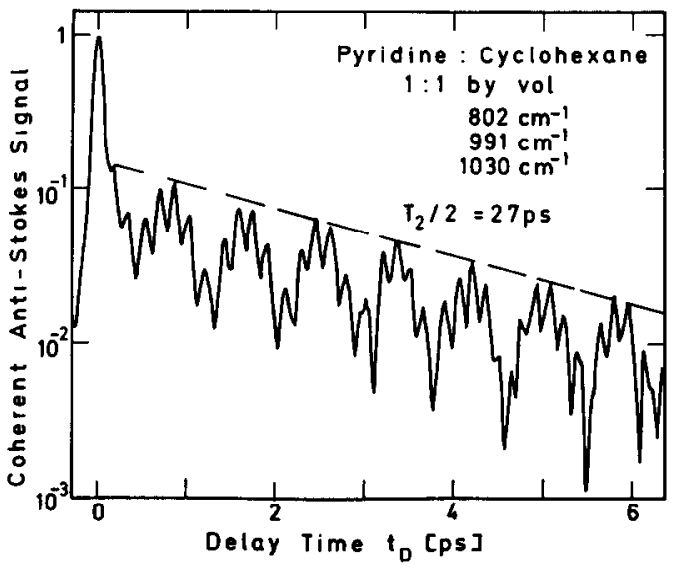

Fig. 4. In the mixture of pyridine and cyclohexane three modes at 1030,991 and $802 \mathrm{~cm}^{-1}$ are excited simultaneously, giving a pronounced beating with beat periods down to $145 \mathrm{fs}$ or beat frequencies up to $6.84 \mathrm{THz}$.

a mixture of cyclohexane and pyridine (1:1 by volume). In this case three strong vibrational modes with similar $T_{2}$ values beat together: one cyclohexane mode at $802 \mathrm{~cm}^{-1}$ and two pyridine modes at 991 and $1030 \mathrm{~cm}^{-1}$. In fig. 4 we find a rich beating structure originating from the interference of the three modes. A Fourier transformation of the measured time dependence of the signal decay was made. We found three peaks in the Fourier spectrum at 39, 189, and $228 \mathrm{~cm}^{-1}$, which correspond to the frequency differences between the three modes excited in our mixture. We point to the very large beat frequency of $228 \mathrm{~cm}^{-1}$ or $\delta \nu=6.84 \mathrm{THz}$

Additional experiments were carried out with a series of molecules. We found various beating structures, in some cases containing even higher beat frequencies of more than $10 \mathrm{THz}$.

\section{Conclusion}

We have presented the first application of femtosecond pulses to coherent Raman spectroscopy. We are able clearly to resolve beating phenomena between different vibrational modes of the molecules comprising the liquid. Beat frequencies as high as $10 \mathrm{THz}$ were observed. These experiments allow us to determine frequency differences between vibrational modes with high precision by timcdomain measurements.

\section{References}

[1] A. Laubereau and W. Kaiser, Rev. Mod. Phys. 50 (1978) 607;

A. Penzkofer, A. Laubereau and W. Kaiser, Progr. Quantum Electron. 6 (1979) 55.

[2] D. von der Linde, A. Laubereau and W. Kaiser, Phys. Rev. Letters 26 (1971) 954

[3] S. Velsko, J. Trout and R.M. Hochstrasser, J. Chem. Phys. 79 (1983) 2114; G.M. Gale, P. Guyot-Sionnest and W.Q. Zheng, Opt. Commun. 58 (1986) 395.

[4] A. Laubereau, G. Wochner and W. Kaiser, Phys. Rev. A13 (1976) 2212;

W. Zinth, H.J. Polland, A. Laubereau and W. Kaiser, Appl. Phys. B26 (1981) 77.

[5] W. Zinth, Opt. Commun. 34 (1980) 479.

[6] W. Zinth, M.C. Nuss and W. Kaiser, Chem. Phys. Letters 88 (1982) 257

[7] W. Zinth, M.C. Nuss and W. Kaiser, Phys. Rev. A30 (1984) 1139.

[8] H. Graener and A. Laubereau, Opt. Commun. 54 (1985) 141.

[9] R.L. Carman, I. Shimizu, C.S. Wang and N. Bloembergen, Phys. Rev. A2 (1970) 60.

[10] J. Dobler, H.H. Schulz and W. Zinth, Opt. Commun. 57 (1986) 407.

[11] D.A. Long, F.S. Murfin and E.L. Thomas, Transactions Faraday Soc. 59 (1963) 12.

[12] W. Zinth, A. Laubereau and W. Kaiser, Opt. Commun. 26 (1978) 457 